\title{
Bioindicadores de la calidad del agua en áreas con restauración ecológica de la quebrada La Colorada, Villa de Leyva, Boyacá.
}

Bioindicators of water quality in areas with ecological restoration of the quebrada La Colorada, Villa de Leyva, Boyacá.

ZULMA EDELMIRA ROCHA GIL

Bióloga

Magíster en Ciencias Ambientales

Grupo de Investigación Gestión Ambiental

Universidad de Boyacá, Colombia zerocha@uniboyaca.edu.co

\section{LUZ ÁNGELA CUELLAR RODRÍGUEZ}

Lic. Biología y Educación Ambiental PhD. en Ciencias Biológicas Grupo de Investigación Gestión Ambiental

Universidad de Boyacá, Colombia luzcuellar@uniboyaca.edu.co

JESSICA LIZETH VARGAS RODRÍGUEZ

Estudiante Ingeniería Ambiental Grupo de Investigación Gestión Ambiental

Universidad de Boyacá, Colombia jesroqriguez@uniboyaca.edu.co

Recibido: 20/05/2015

\section{XIOMARA DÍAZ SOLER}

Estudiante Ingeniería Ambiental Grupo de Investigación Gestión Ambiental

Universidad de Boyacá, Colombia xsoler@uniboyaca.edu.co

Aceptado: 14/10/2015 


\section{RESUMEN}

El presente estudio evaluó la calidad ecológica de la Quebrada La Colorada en diferentes períodos pluviométricos de los años 2014 y 2015, en el municipio de Villa de Leyva, Boyacá - Colombia, a través de caracterización de macroinvertebrados, seguimiento de parámetros fisicoquímicos y aplicación de índices bióticos. Se seleccionaron 2 estaciones de muestreo en el sistema, una en áreas de bosque tropical restauradas luego de presentar episodios de incendios forestales, y la otra en zonas no restauradas aledañas. En cada estación de muestreo se capturaron macroinvertebrados con una red Surber a lo largo de 100 metros. Los resultados muestran diferencias significativas en la composición de la comunidad bentónica asociada a las áreas restauradas con la presencia de 4 clases (Gastropoda, Oligochaeta, Turbellaria e Insecta); 10 órdenes, 15 familias y un total de 18 morfotipos de macroinvertebrados, en la zona control sin restauración se encontraron organismos de la 2 clases (Gastropoda e Insecta), 5 órdenes, 6 familias y un total de 7 morfotipos de macroinvertebrados y se presentan 6 géneros comunes en las 2 estaciones. Lo anterior se asocia a cambios físico-químicos del sistema, variación de microhábitats y condiciones pluviométricas de las épocas de muestreo. Los análisis de diversidad muestran diferencias significativas entre las 2 estaciones; al igual que los valores de dominancia. Los valores de similitud relacionan una alta variabilidad en la composición de especies entre estaciones, los especímenes comunes entre estaciones presentan condiciones de hábitat asociados a aguas limpias y rápidas con presencia de material autóctono, además de poca tolerancia a la contaminación orgánica.

Palabras clave: Bioindicadores, restauración ecológica, macroinvertebrados y bosque andino.

\footnotetext{
ABSTRACT

The present study evaluated the ecological quality of the Quebrada La Colorada, in the municipality of Villa de Leyva, Boyacá - Colombia, in different rainfall periods of 2014 and 2015, through characterization of macroinvertebrates, the monitoring of physicochemical parameters and application of biotic indexes. Two sampling stations were selected in the system, one in tropical forest area restored
} 
after episodes of forest fires, and another one surrounding not restored areas. In each sampling station macroinvertebrates were captured in a Surber Net along 100 meters. The results show significant differences in the composition of the benthic community associated to the restored areas with the presence of 4 classes (Gastropoda, Oligochaeta, Turbellaria and Insecta), 10 orders, 15 families and a total of 18 morphotypes of macroinvertebrates. In the control areas without restoration, organisms of two classes were found (Gastropoda and Insecta), 5 orders, 6 families and a total of 7 morphotypes of macroinvertebrates, also 6 common genera are presented in 2 seasons. The situation presented is associated with physical and chemical changes of the system, variation of microhabitats and pluviometric conditions during sampling times. Analyses of diversity showed significant difference between the 2 stations; as well as the values of dominance. The similarity values link a high variability in the composition of species among stations; common specimens between seasons present conditions of habitat associated with fast and clean waters, with presence of autochthonous material, as well as a little tolerance to organic pollution.

Keywords: Bio-indicators, ecological restoration, macroinvertebrates, Andean Forest. 


\section{INTRODUCCIÓN}

Los estudios orientados al seguimiento de procesos de restauración ecológica implican diferentes aspectos como recuperar la funcionalidad y estructura del sistema natural, integración de flujos bióticos y abióticos, entre otros (Vargas, 2007). En los últimos años en Colombia ha venido creciendo el interés por la restauración ecológica luego de diferentes procesos de afectación ambiental, entre ellos los incendios forestales, esto ha permitido la generación de proyectos desarrollados en ecosistemas terrestres y acuáticos (Vargas, 2007). Estos procesos han sido asociados recientemente a la oferta de servicios ambientales en ecosistemas acuáticos, de acuerdo con Little y Lara (2010) para conservar o mantener cuencas en estados naturales o intervenidos, mejorando aspectos como prácticas de manejo de suelo, composición, densidad y tamaño de bosques.

En este mismo sentido, otros estudios que han evaluado acciones de restauración ecológica sobre la recuperación de diversos servicios ecosistémicos, demuestran un impacto positivo de tales acciones en servicios como la diversidad biológica, la fertilidad de suelos y la capacidad productiva de diferentes ecosistemas (Rey-Benayas et al. 2009). Dentro de este contexto, las relaciones conocidas entre cobertura boscosa de las cuencas y la provisión de agua son la base para plantear la recuperación de este servicio ecosistémico. De esta manera, los principios y prácticas de la restauración definidos por SER (2009) permiten relacionar fundamentos conceptuales para emprender proyectos en cuencas perturbadas, entendidas como ecosistemas a restaurar.

De la misma manera, en la actualidad los cuerpos de agua son considerados sensibles a modificaciones antrópicas, debido a que se han vuelto depósitos de desechos causando la reducción de comunidades bióticas (Lozano, 2005), además la fragmentación y pérdida de hábitats son la fuente del remplazo de bosques nativos por ecosistemas antrópicos (Cardona, 2004), reduciendo las posibilidades de restauración, además de la pérdida de biodiversidad y variabilidad genética, la cual se ha contribuido a la restauración ecológica donde un ecosistema degradado alcanza parte de su estructura y función debido al proceso de sucesión ecológica. (Cardona, 2004 cita a Sorzano, 2001).

Cabe resaltar que no simplemente la contaminación antrópica influye en la distribución y crecimiento de las especies, por lo que se deben considerar factores climáticos, geográficos y simbióticos que alteran una comunidad (Giacometti, 2006). Las cuencas están caracterizadas por las interacciones geomorfológicas, hidrológicas y biológicas que determinan las comunidades presentes en sus ríos, observando los cambios graduales en composición taxonómica y características físicas y químicas aparecen dos conceptos importantes: continuo del río referente a la distribución de especies en un río desde su 
nacimiento hasta su desembocadura (Vannote, 1980). De acuerdo a esto; en los ríos se pueden encontrar microorganismos bioindicadores los cuales dependen de las diferentes características que se presentan en el agua, tales como la disponibilidad de luz, la oferta de alimento, la presencia de depredadores, entre otros; estos factores determinan la presencia de ciertas familias de organismos en unos sitios y su reemplazo por familias diferentes en otros sitios, pero que cumplen la misma función en el sistema (Bernal, et al 2005). Por lo tanto, para poder evaluar el deterioro ambiental de un ecosistema acuático debido a un contaminante es necesaria la selección de una comunidad bioindicadora de calidad de agua y el conocimiento previo de la biota que caracteriza la zona de estudio. Uno de los grupos de los organismos acuáticos más utilizados para el monitoreo, son los macroinvertebrados de la comunidad bentónica (Zúñiga, 1993).

Por lo tanto, para la determinación e identificación de la calidad de los sistemas hídricos es importante tener en cuenta las características fisicoquímicas y biológicas que se presentan en dichos cuerpos de agua, dentro de las características físicas es importante tener en cuenta la temperatura y la densidad del agua, siendo estos parte de uno de los factores ambientales con gran importancia para el análisis y descripción de los cuerpos de agua, esto se demuestra principalmente en el desempeño que realizan estos parámetros en los numerosos procesos de regulación que se llevan a cabo en los ecosistemas acuáticos (Esteves, 1988).

De otra parte, una de las herramientas de seguimiento de estado de los ecosistemas acuáticos es la aplicación de índices de diversidad biológica con macroinvertebrados como el de Shannon - Wiener $\left(\mathrm{H}^{\prime}\right)$ y dominancia de Simpsom (D). Finalmente, con el objetivo de comparar el grado de similitud entre las estaciones se empleó el coeficiente de Jaccard (J) (Castellanos y Serrato 2008), además de la implementación de estas herramientas es importante tener en cuenta determinados parámetros físico-químicos para realizar un análisis y comparación de acuerdo a los valores obtenidos; entre estos se encuentran el pH, del cual dependen fenómenos como la respiración y la fotosíntesis que se presentan en los cuerpos de agua, en donde su valor permite identificar las variaciones de niveles de oxígeno, relacionando directamente una alta o baja presencia de macroinvertebrados, puesto que muchos de estos no resisten cambios fuertes. De otra parte, el nitrógeno hace parte fundamental de los seres vivos en los sistemas acuáticos dado que dependiendo de su valor, se puede identificar fácilmente indicios de contaminación, además de esto es importante resaltar que los elevados niveles de nitrógeno en este medio, traen consigo procesos de eutrofización, provocando una fuerte reducción en la diversidad de especies. De igual manera, el fósforo es otro elemento importante que tiene un mayor efecto en el proceso de eutrofización, favoreciendo el crecimiento de fitoplancton y afectando la estructura y el funcionamiento del ecosistema acuático. (Roldán, 2003). 
En este mismo sentido (Roldán, 1999) indica que no todos los organismos acuáticos podrán ser tomados como bioindicadores, debido a que presentan diferentes adaptaciones evolutivas en condiciones ambientales variadas y a límites de tolerancia en determinadas alteraciones, generando cambios en las características de los diferentes grupos que podrán ser considerados como organismos sensibles (Ephemeroptera, Plecoptera, Trichoptera), por no soportar variaciones en la calidad del agua, mientras que organismos tolerantes (Chironómidae, Oligoquetos), son característicos de agua contaminada por materia orgánica (Roldán, 1999). Cuando los cambios en los parámetros físico-químicos son críticos, los organismos sensibles mueren y su lugar es ocupado por los organismos tolerantes (Alba-Tercedor, 1996), de tal forma que los cambios de la estructura y composición de las comunidades bióticas puede ser utilizada para identificar y evaluar los grados de contaminación de un ecosistema acuático.

Por otro lado, en el país se ha avanzado en varias regiones en temas como la limnología fisicoquímica de ríos, lagos y embalses, lo cual esto ha contribuido en el mejoramiento de los ecosistemas acuáticos; sin embargo, hoy en día falta conocer varios grupos (Roldán \& Ramírez, 2008). Según (Roldán, 2003) el BMWP (Biological Monitoring Working Party) para Colombia, se ha modificado y adaptado a partir de los estudios de macroinvertebrados establecidos en algunas regiones del país, siendo la medida de tolerancia - intolerancia, dando una puntuación de BMWP y ASPT (Average Score Per Taxon), dependiendo de la asignación de valores establecidos para las familias y los valores de riqueza, donde el índice se genera a partir de la presencia de familias sensibles o tolerantes a la contaminación del agua (Arango, 2008), siendo una herramienta para caracterizar las aguas superficiales.

En Colombia también los estudios de calidad de agua se han basado históricamente en la caracterización de parámetros fisicoquímicos y microbiológicos, no se tienen en cuenta otros componentes de los ecosistemas como la comunidad de los macroinvertebrados (Luna, 2009), los cuales se utilizan como testigos biológicos del nivel de deterioro ambiental de las corrientes superficiales, puesto que reflejan las condiciones y los cambios ecológicos que ocurren en el sistema (Arango, 2008), donde estos juegan un papel importante siendo el principal componente animal de los sistemas lóticos, además estos representan una función importante en la red trófica del sistema dulce acuícola y aceleran la descomposición de detritos además de contribuir al reciclaje de nutrientes (Castellanos, cita a Wade, 1989.). De acuerdo a lo anterior, los macroinvertebrados ofrecen diversas ventajas y amplia distribución, adaptación a diferentes variables físico-bióticas, simplicidad metodológica, rapidez de los resultados y una retrospectiva a los eventos de contaminación (Roldán, 1999). Estos organismos que habitan en los cuerpos de agua presentan unos límites de tolerancia que varían de acuerdo a las alteraciones que se encuentran en organismos sensibles que no soportan las condiciones impuestas siendo intolerantes, mientras que otros tolerantes no se ven afectados; es decir, las variaciones repentinas puede ser presencia de cualquier tipo de contaminación, además de proporcionar la ventaja de evidenciar las condiciones dadas tiempo atrás antes de la toma de muestras. (Alba - Tercedor, 1996). De tal modo que el uso de macroinvertebrados revelan la manifestación de cambios de diversos y complejos organismos propios de aguas limpias a simples y de poca diversidad, con organismos

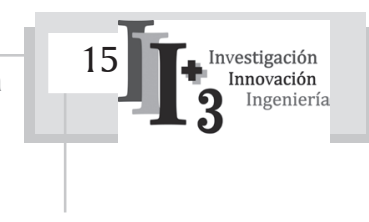


de aguas contaminadas, donde el $\mathrm{pH}$, conductividad, concentración de oxígeno y altitud son variables que dependen de ellos para sobrevivir. (Terneus, 2012 cita a Jill, S).

En el departamento de Boyacá se han adelantado algunos estudios para la determinación de bioindicadores desde el punto de vista biológico y ecológico, por ejemplo en el año 2005 se adelantó un estudio en la Cuenca Alta del Río Chicamocha, en el cual se determinaron los índices de calidad según las relaciones reportadas en la Limnología Colombiana de Ramírez et al, (1998). A partir de la determinación de estos índices se realizó un análisis sencillo en el que se clasificó la calidad del agua en buena, mala y regular y de los resultados fisicoquímicos, se relacionaron los usos que se le deben dar a la corriente en los diferentes tramos analizados, según lo establecido en el Decreto 1594 del año 1984 sobre usos del agua y vertimientos (Rocha, 2007). A nivel regional en la Quebrada La Colorada del municipio de Villa de Leyva, las actividades antrópicas desarrolladas, como captaciones de suministro de agua, adecuación de terrenos para la ganadería, agricultura y los distintos incendios que han afectado la Quebrada, traen como consecuencia el deterioro de la oferta ambiental, debido a que alteran la composición y funcionamiento ecológico de los organismos que viven en el sistema acuático, en donde varios de estos organismos tienen adaptaciones únicas a microhábitats específicos, fuentes de alimentación, entre otras, mientras que otras son cosmopolitas y aptas para sobrevivir a una amplia gama de condiciones (Ramírez, et al., 2008). Además, otro factor importante en la dinámica de este sistema, es el transporte de las partículas disueltas y la materia orgánica que se produce a lo largo del ecosistema, generando una estrecha relación entre el ecosistema terrestre y la quebrada. Por otro lado, la vegetación ribereña ejerce un papel de gran importancia en la cadena trófica, aportando al flujo natural de la materia y energía, cooperando con el proceso del ciclo de nutrientes especialmente del nitrógeno y fósforo, disponibilidad de sustratos orgánicos y la generación de hábitats. (Vargas, 2007).

\section{METODOLOGÍA}

Área de estudio:

La cuenca de la quebrada La Colorada pertenece al municipio de Villa de Leyva, Departamento de Boyacá y está ubicada en el área de amortiguación de bosque andino del Macizo de Iguaque, perteneciente a la cadena montañosa plegada en rocas sedimentarias (areniscas). Presenta una longitud aproximada de $750 \mathrm{~m}$. desde su naciente hasta la desembocadura. El Nacimiento atmosféricamente se encuentra en una zona húmeda, está ubicado a los $05^{\circ} 38^{`} 45.9^{\prime \prime} \mathrm{N}$ y $0.73^{\circ} 30^{`} 30^{\prime \prime}$ W a 2850 m.s.n.m. y desemboca en el río Leyva a una altitud de 2600 m.s.n.m. La región presenta un ciclo hidrológico bimodal, con una precipitación media anual de $953 \mathrm{~mm}$, con dos períodos de baja precipitación que comprende los meses de diciembre a marzo, y dos períodos de alta precipitación comprendidos entre (abril y noviembre). (Plan de Manejo Santuario de Fauna y Flora de Iguaque, 2006). Las estaciones de muestreo se ubicaron en las zonas identificadas como impactadas con presencia de ceniza, aumento 
de sólidos suspendidos y el incremento de color y turbiedad según el Estudio de Impacto Ambiental del Macizo de Iguaque realizado en el año 2010.

El uso del suelo en el sector es principalmente de protección, debido a que en la zona se localiza la bocatoma para suministro parcial de agua a la Planta de Tratamiento de Agua Potable de la Empresa de Servicios Públicos de Villa de Leyva "Esvilla ESP", además presenta un área de bosque andino con fragmentación, debido a los disturbios generados frecuentemente por incendios forestales. El área objeto de estudio se encuentra en la cuenca media de la Quebrada La Colorada, en donde se han realizado procesos de restauración ecológica no asistida (proceso de revegetalización con especies nativas), a partir de la especie Quercus humboldtii como parte de los proyectos de cooperación internacional para recuperar la zona, realizados por la ONG ECOHUMANA (Fundación para el Desarrollo Humano Integral Sostenible).

\section{Muestreo y análisis}

Se realizaron 4 eventos muestreales y se seleccionaron 2 estaciones de muestreo en el sistema, una en áreas de bosque tropical restauradas y la otra en zonas no restauradas aledañas, en cada estación de muestreo se capturaron macroinvertebrados con una red Surber de $30 * 30 \mathrm{~cm}$. y $250 \mu \mathrm{m}$ de diámetro de poro a lo largo de 100 metros, abarcando $1 \mathrm{~m}^{2}$ por estación. Los individuos colectados fueron depositados en frascos plásticos debidamente rotulados, con alcohol al 70\% para su preservación, los muestreos se realizaron en los períodos de transición de lluvias y sequía, teniendo en cuenta los registros pluviométricos históricos, en los respectivos meses de aguas bajas (julio y diciembre de 2014) y aguas altas (octubre de 2014 y marzo de 2015).

En el laboratorio se procedió a la limpieza y separación de los organismos mediante un estereoscopio Olympus SZ40 y un microscopio Olympus CH30. Los organismos recolectados se determinaron hasta el mínimo nivel de resolución taxonómico posible mediante claves y descripciones de Roldán (1996), Roldán (2003), Manzo (2005), Domínguez et al. (2006), Merrit \& Cummins (2008), Domínguez \& Fernández (2009).

Con el fin de describir el hábitat, en cada estación se registraron in situ los parámetros de temperatura, $\mathrm{pH}, \mathrm{y}$ conductividad, empleando una sonda multiparámetro portátil marca SCHOTT de la serie L1281022 y, Nitratos $\left(\mathrm{NO}_{2}\right)$, Nitritos $\left(\mathrm{NO}_{3}\right)$ y Amonio $\left(\mathrm{NH} 4^{+}\right)$, Fosfatos $\left(\mathrm{PO}_{4}^{-}\right)$y Oxígeno Disuelto mediante Kid rápido de aguas de la marca Aqua Merck.

Para analizar la comunidad de macroinvertebrados en las 2 estaciones de muestreo se emplearon los parámetros biológicos de riqueza de especies de Margalef $(\mathrm{R})$, diversidad biológica de Shannon - Wiener $\left(\mathrm{H}^{\prime}\right)$ y dominancia de Simpsom $(\lambda)$. Finalmente, con el objetivo de comparar el grado de similitud entre las estaciones se empleó el coeficiente de Jaccard (J) y se realizó un dendograma de disimilitud como método de representación gráfica, empleando el programa estadístico SPSS versión 19. 


\section{RESULTADOS Y DISCUSIÓN}

Para las dos estaciones de la quebrada La Colorada se registraron valores promedio de temperatura entre los $17.5^{\circ} \mathrm{C}$ y $18.5^{\circ} \mathrm{C}$ y pH entre 6.5 a 7 , observándose poca variabilidad en estos parámetros en las áreas evaluadas con y sin restauración ecológica. En cuanto a concentración de oxígeno disuelto presentó una variación significativa en las dos áreas de estudio, así como también en los diferentes períodos de muestreo, registrando un valor máximo de $2.25 \mathrm{mg} / \mathrm{O}_{2}$ en el área restaurada en los meses de julio y diciembre, los valores mínimos se presentaron en la zona no restaurada con un registro de $1.7 \mathrm{mg} / \mathrm{O} \mathrm{O}_{2}$ en los meses de diciembre y marzo que corresponden a épocas de precipitaciones altas (Ver Tabla 1.), razón por la cual se asocian estos cambios al aporte de materia orgánica de origen autóctono y a las condiciones locales predominantes de zonas de rápidos en el área restaurada y algunas áreas de cubeta en la no restaurada.

En relación con las diferentes formas disponibles de nitrógeno en el sistema, se determinó un valor máximo de nitratos de $10 \mathrm{mg} / \mathrm{NO}_{3}$ en la zona no restaurada en el mes de octubre, cabe destacar que el área, está influenciada por actividades pecuarias y presenta mayor pendiente, lo que posibilita la escorrentía de nutrientes en la época de lluvias. Los registros de nitritos y amonio reportaron valores entre $0 \mathrm{mg} / \mathrm{l}$ y $0.16 \mathrm{mg} / \mathrm{l}$ respectivamente para los meses de julio y diciembre en las épocas de aguas bajas, probablemente este aumento se relaciona con descargas de aguas residuales o presencia eventual de contaminantes de origen animal (Ver Tabla 1.).

El registro de fosfatos presentó una concentración constante $0.25 \mathrm{mg} / \mathrm{l}$, excepto para el mes de octubre en donde se reportó $0.5 \mathrm{mg} / \mathrm{l}$, esto debido a posibles efectos residuales de la presencia de ceniza en el agua, producto de los incendios forestales del año 2010, lo cual se relaciona con el incremento de concentración de fósforo y amonio.

De otra parte, la caracterización físico-química de la quebrada La Colorada refleja un comportamiento típico de sistemas interandinos, con bajos niveles de conductividad asociados posiblemente al proceso de disolución de las rocas y limitado aporte de sólidos de estas áreas geológicas. 


\begin{tabular}{|c|c|c|c|c|c|c|c|c|}
\hline \multirow{2}{*}{ PARÁMETRO } & \multicolumn{2}{|c|}{ JULIO } & \multicolumn{2}{|c|}{ OCTUBRE } & \multicolumn{2}{|c|}{ DICIEMBRE } & \multicolumn{2}{|c|}{ MARZO } \\
\hline & P1 & P2 & P1 & P2 & P1 & P2 & P1 & P2 \\
\hline Temperatura del agua $\left({ }^{\circ} \mathrm{C}\right)$ & $\begin{array}{l}17.5- \\
18.5\end{array}$ & $\begin{array}{l}17.5- \\
18.5\end{array}$ & $\begin{array}{l}17.5- \\
18.5\end{array}$ & $\begin{array}{l}17.5- \\
18.5\end{array}$ & $\begin{array}{l}17.5- \\
18.5\end{array}$ & $\begin{array}{l}17.5- \\
18.5\end{array}$ & $\begin{array}{l}17.5- \\
18.5\end{array}$ & $\begin{array}{l}17.5- \\
18.5\end{array}$ \\
\hline Nitritos $\left(\mathrm{mg} / \mathrm{l} \mathrm{NO}_{2}\right)$ & 0 & 0 & 0 & 0 & 0 & 0 & 0 & 0 \\
\hline Nitratos(mg/l NO$\left.{ }_{3}\right)$ & 0 & 0 & 5.25 & 10 & 0 & 0 & 0 & 0.5 \\
\hline Amonio $\left(\mathrm{mg} / \mathrm{l} \mathrm{NH}_{4}^{+}\right)$ & 0.08 & 0.16 & 0 & 0 & 0.08 & 0.16 & 0.08 & 0 \\
\hline $\begin{array}{l}\text { Oxígeno Disuelto } \\
\left(\mathrm{mg} / \mathrm{l} \mathrm{O}_{2}\right)\end{array}$ & 2.25 & 2.0 & 1.75 & 2.7 & 2.25 & 1.7 & 1.8 & 1.7 \\
\hline Fosfatos (mg/l $\left.\mathrm{PO}_{4}^{-3}\right)$ & 0.25 & 0.25 & 0.5 & 0.25 & 0.25 & 0.25 & 0.125 & 0.25 \\
\hline $\mathrm{pH}$ & 6.7 & 7 & 6.75 & 7 & 6.5 & 7 & 6.75 & 7 \\
\hline Conductividad $(\mu \mathrm{S} / \mathrm{cm})$ & - & - & 54.4 & 52.3 & - & - & 62.5 & 52.4 \\
\hline
\end{tabular}

Tabla 1. Parámetros físico-químicos quebrada La Colorada. (Julio, octubre y diciembre de 2014 - Marzo de 2015).

El listado general de taxones registrados en las áreas con y sin restauración se presenta en las Tablas 2 y 3 , con un total de 172 individuos de macroinvertebrados acuáticos, pertenecientes a 19 morfoespecies, 16 familias y 10 órdenes.

Los valores de los parámetros de diversidad alfa son presentados en la Tabla 3. El orden más diverso fue hemiptera con 5 morfoespecies, alcanzando una representatividad del 63,37\% de los taxones, también presentó la mayor abundancia con 109 individuos. El promedio de la riqueza de especies presentó una variabilidad entre 4.08 y 8.16. En general, el número de taxones fue mayor en la estación uno, presentando diferencias con relación a la estación dos. La variabilidad de la riqueza específica en la cuenca, muestra que los valores más altos se encuentran en el sector restaurado. En la estación con área de influencia no restaurada los valores son menores, variando de 2 a 8 especies.

\begin{tabular}{lllll}
\hline \hline \multicolumn{1}{c}{ PHYLUM } & \multicolumn{1}{c}{ CLASE } & \multicolumn{1}{c}{ ORDEN } & \multicolumn{1}{c}{ FAMILIA } & \multicolumn{1}{c}{ GÉNERO } \\
\hline \hline Mollusca & Gastropoda & Basommatophora & Ancylidae & Ferrissia sp. \\
Annelida & Oligochaeta & Haplotaxida & Tubificidae & Actedrilus $\mathrm{sp}$. \\
Platyhelminthes & Turbellaria & Tricladida & Planariidae & Dugesia sp. \\
\hline \hline
\end{tabular}




\begin{tabular}{|c|c|c|c|c|}
\hline PHYLUM & CLASE & ORDEN & FAMILIA & GÉNERO \\
\hline \multirow{12}{*}{ Arthropoda } & \multirow{12}{*}{ Insecta } & Coleoptera & $\begin{array}{l}\text { Dryopidae } \\
\text { Ptilodactylidae }\end{array}$ & $\begin{array}{l}\text { Pelonomus sp. } \\
\text { Anchytarsus sp. }\end{array}$ \\
\hline & & \multirow[t]{2}{*}{ Ephemeroptera } & Baetidae & $\begin{array}{l}\text { Baetodes sp. } \\
\text { Dactylobaetis sp. }\end{array}$ \\
\hline & & & Leptophlebiidae & Thraulodes sp. \\
\hline & & \multirow{4}{*}{ Hemiptera } & Veliidae & $\begin{array}{l}\text { Stridulivelia sp. } \\
\text { Microvelia sp. }\end{array}$ \\
\hline & & & & Rhagovelia sp. \\
\hline & & & Mesoveliidae & Mesoveloidea sp. \\
\hline & & & Guerridae & Limnogonus sp. \\
\hline & & Diptera & Ceratopogonidae & Stilobezzia sp. \\
\hline & & Odonata & Coenagrionidae & Acanthagrion sp. \\
\hline & & Plecoptera & Perlidae & Anacroneuria sp. \\
\hline & & \multirow{2}{*}{ Trichoptera } & Glossosomatidae & Mortoniella sp. \\
\hline & & & Leptoceridae & Atanatolica sp. \\
\hline
\end{tabular}

Tabla 2. Relación de taxas encontrados en áreas con restauración ecológica de la quebrada La Colorada.

De acuerdo con la Tabla anterior, el área de estudio con restauración ecológica registró un total de 128 morfotipos, pertenecientes a 10 órdenes. El orden más diverso fue Hemiptera con 114 morfoespecies, 3 familias y 5 géneros, alcanzando una representatividad del 64,04\% de los taxones.

\begin{tabular}{cclll}
\hline \hline PHYLUM & CLASE & \multicolumn{1}{c}{ ORDEN } & \multicolumn{1}{c}{ FAMILIA } & \multicolumn{1}{c}{ GÉNERO } \\
\hline \hline Mollusca & Gastropoda & Basommatophora & Ancylidae & Ferrissia sp. \\
& & Coleoptera & Ptilodactylidae & Anchytarsus sp. \\
& & Ephemeroptera & Baetidae & Dactylobaetis sp. \\
& & Trichoptera & Helicopsychidae & Helicopsyche sp. \\
Arthropoda & Insecta & & Mesoveliidae & Mesoveloidea sp. \\
& & Hemiptera & Veliidae & Microvelia sp. \\
& & & & Rhagovelia sp. \\
\hline \hline
\end{tabular}

Tabla 3. Relación de taxas encontradas en áreas sin restauración ecológica de la quebrada La Colorada. 


\begin{tabular}{|c|c|c|c|}
\hline \multirow[t]{2}{*}{ FECHA } & \multirow[t]{2}{*}{ VARIABLE } & \multicolumn{2}{|c|}{ ESTACIÓN } \\
\hline & & P1 & P2 \\
\hline \multirow[t]{5}{*}{ JULIO } & $\mathrm{n}$ & 19 & 8 \\
\hline & S & 0.14 & 0.25 \\
\hline & $\mathrm{H}^{\prime}$ & 1.58 & 1.07 \\
\hline & $\lambda$ & 0.28 & 0.43 \\
\hline & $\mathrm{R}$ & 4.08 & 1.92 \\
\hline \multirow[t]{5}{*}{ MARZO } & $\mathrm{n}$ & 41 & 18 \\
\hline & $S$ & 0.14 & 0.5 \\
\hline & $\mathrm{H}^{\prime}$ & 1.67 & 0.45 \\
\hline & $\lambda$ & 0.21 & 0.72 \\
\hline & $\mathrm{R}$ & 9.16 & 5.53 \\
\hline \multirow[t]{5}{*}{ OCTUBRE } & $\mathrm{n}$ & 36 & 19 \\
\hline & $S$ & 0.13 & 0.33 \\
\hline & $\mathrm{H}^{\prime}$ & 1.03 & 0.99 \\
\hline & $\lambda$ & 0.57 & 0.40 \\
\hline & $\mathrm{R}$ & 7.81 & 5.43 \\
\hline \multirow[t]{5}{*}{ DICIEMBRE } & $\mathrm{n}$ & 34 & 3 \\
\hline & $S$ & 0.20 & 1 \\
\hline & $\mathrm{H}^{\prime}$ & 1.23 & 0 \\
\hline & $\lambda$ & 0.39 & 1 \\
\hline & $\mathrm{R}$ & 8.22 & 1.82 \\
\hline
\end{tabular}

Tabla 4. Individuos (n), abundancia (S), diversidad ( $\left.H^{\prime}\right)$, dominancia $(\lambda)$ y riqueza $(R)$ de macroinvertebrados acuáticos en los eventos muestreales de la quebrada La Colorada.

El promedio de individuos en todos los muestreos varió de 27 a 59, la estación que reportó mayor número de individuos fue el área restaurada con un total de 124 y el menor número en la zona no restaurada con 48 especímenes, probablemente por la potencialidad de hábitats asociados a la vegetación establecida en las áreas restauradas. 
La mayor diversidad alfa se observó para esta zona con un valor de 1,67 como se registra en la Tabla 4. El valor máximo de dominancia se evidenció en la zona no restaurada con un valor de 1 en dos de los cuatro eventos muestreales. El promedio de la riqueza de especies entre las dos estaciones presentó mayor variabilidad con registros entre 1,82 y 9,16. En general, el número de taxones fue mayor en la estación uno, presentando diferencias con relación a la estación 2.

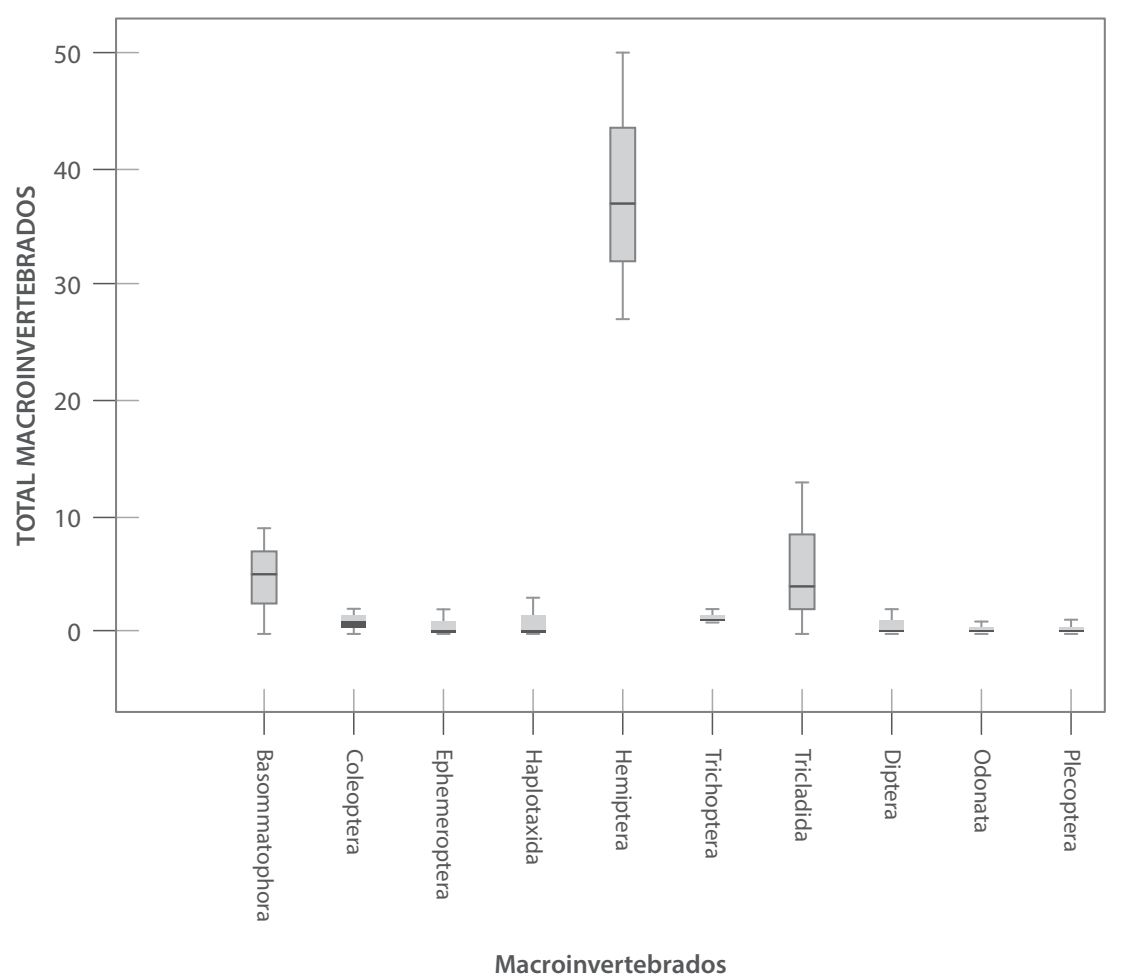

Figura 1. Total de órdenes de macroinvertebrados en los eventos muestreales en la quebrada La Colorada.

En la Figura 1 se muestra la contribución porcentual de cada orden. El Orden Hemiptera presentó la mayor abundancia con 77 individuos y los órdenes Plecoptera y Odonata presentaron la menor abundancia, con 1 individuo.

Los valores de similitud son mostrados en la Figura 2, indican una alta variabilidad en la composición de especies, mostrando que los sitios de muestreo de la Quebrada pueden presentar una composición taxonómica de macroinvertebrados muy diferentes. Según Heino et al., (2004) la composición de macroinvertebrados en diferentes hábitas es más variable en las pequeñas cabeceras de ríos que en los de mayor orden. Esto puede obedecer a los cambios de sustrato y cobertura de bosque, los 
cuales son factores importantes para los macroinvertebrados acuáticos como lo menciona Hawkins et al., (1982). En consecuencia los resultados hallados reflejan que la Quebrada La Colorada presenta cambios en la estructura de la comunidad de macroinvertebrados acuáticos entre las zonas restauradas y no restauradas.

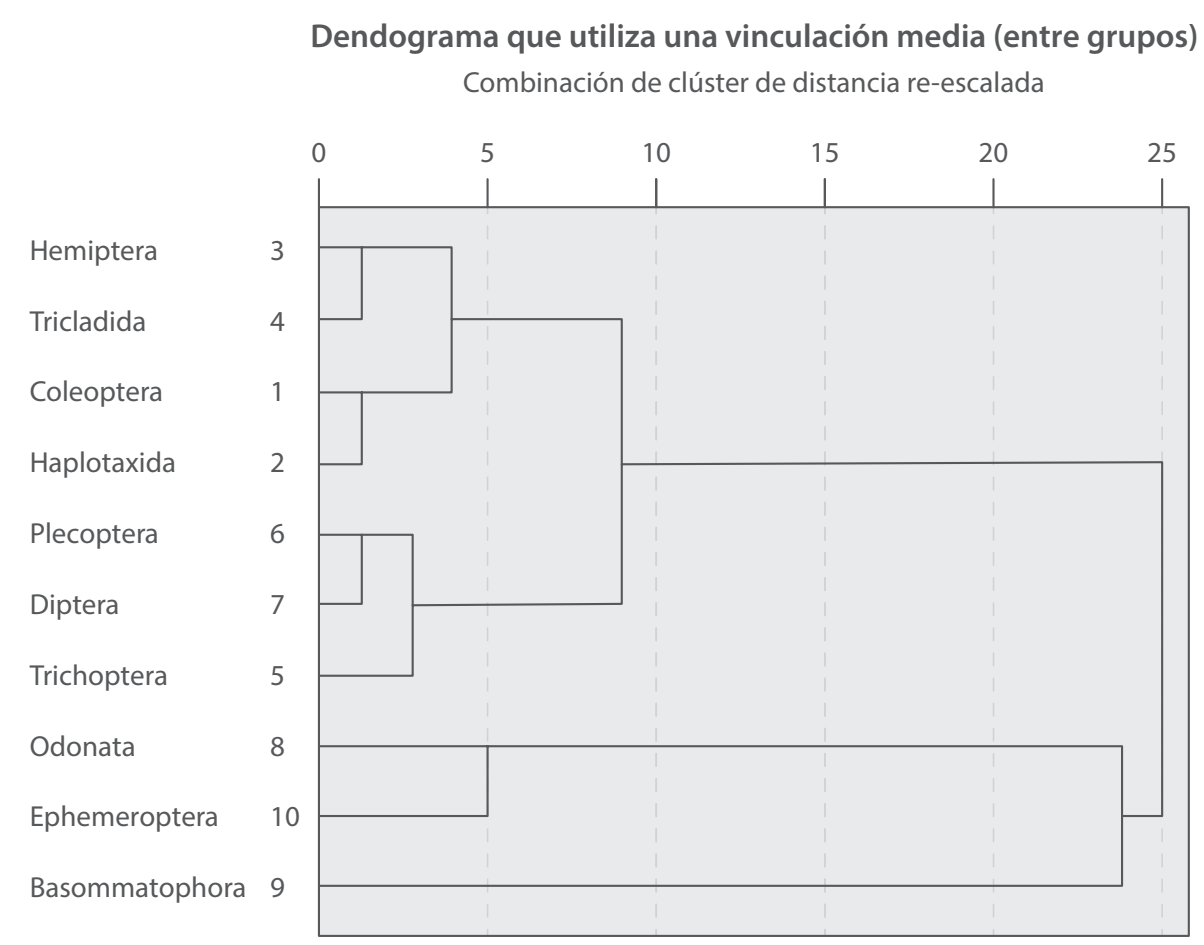

Figura 2. Dendograma de similitud entre órdenes de los eventos muestreales en la quebrada la colorada.

El dendograma de la Figura 2, muestra una mayor similitud asociada a la frecuencia de los órdenes como Hemiptera, Coleoptera y Tricladida y la mayor disimilitud en los órdenes Haplotaxida, Odonata y Basommatophora en las dos estaciones de muestreo. Esto se relaciona con la ecología de estos grupos, se presume mayor disponibilidad de hábitats con sustratos representativos como troncos y hojas en descomposición, grava, piedras, arena y vegetación sumergida y emergente con concentraciones altas, correspondiente a las características encontradas en zona restaurada en las épocas de muestreo.

Los órdenes asociados a disimilitud son característicos de zonas lénticas, poco profundas con abundante vegetación acuática y ligera eutroficación, los cuales se presentaron con mayor frecuencia en los eventos muestreales de la zona no restaurada, esto probablemente a posibles fuentes de contaminación 
en el área de influencia; así como a su poca capacidad de resiliencia por los diferentes procesos de intervención antrópica y efectos residuales de los incendios forestales.

\section{CONCLUSIONES:}

Los análisis de diversidad muestran un valor medio para la zona restaurada de acuerdo a los rangos propuestos por (Margalef, 1983), indicando que la comunidad presenta factores de resistencia ambiental que permiten mantener diferentes niveles tróficos, frente a la zona no restaurada, debido a que esta presentó valores bajos de diversidad.

Teniendo en cuenta lo descrito por (Vannote et al.,1980) en cuanto a la baja diversidad de las comunidades que habitan los nacimientos de ríos por bajas temperaturas y poco aporte de materia orgánica, se puede concluir que a pesar de los disturbios presentados en la cuenca alta de la Quebrada La Colorada, se encontraron diferencias marcadas en los resultados de dominancia, riqueza, similitud y diversidad, relacionándose con los procesos de restauración ecológica realizados en esta zona, lo que permite la formación de nuevos hábitats, aumentando las especies.

Los resultados reflejan que la Quebrada La Colorada presenta cambios en la estructura de la comunidad de macroinvertebrados acuáticos entre las zonas restauradas y no restauradas en las diferentes épocas muestreadas, probablemente también por la variabilidad en parámetros meteorológicos, aporte de materia orgánica de origen autóctono y alóctono y condiciones locales predominantes de zonas de rápidos en el área restaurada y algunas áreas de cubeta en la no restaurada. Al igual estos cambios pueden estar relacionados con las diferentes formas disponibles de nitrógeno en el sistema y aportes de fosfatos por efectos residuales de la presencia de ceniza en el agua producto de los incendios forestales del año 2010. 


\section{REFERENCIAS BIBLIOGRÁFICAS}

Alba - Tercedor, J. (1996). Macroinvertebrados Acuáticos y Calidad de las Aguas de los Ríos. Universidad de granada. Colombia. 84-7840-262-4.

Arango, M. \& Álvarez, L. (2008). Calidad del Agua de las Quebradas la Cristalina y la Risaralda, San Luis, Antioquia. Universidad de Antioquia, Medellín - Colombia. 1794-1237. Vol. 5. Bosque 31(3): 175-178, 2010 Restauración ecológica y provisión de agua.

Bernal, E., García, D., Novoa, A. \& Pinzón, A. (2005). Caracterización de la comunidad de macroinvertebrados de la Quebrada Paloblanco de la cuenca del río Otún (Risaralda, Colombia). 1-15.

Cardona, A. \& Vargas, O. (2004). El Banco de Semillas Germinables de Especies en Dos Bosques Subandinos y su Importancia para la Restauración Ecológica. Reserva Biológica Cachalú Santander- Colombia. 8 (4): 350-360.

Castellanos, P. \& Serrato, C. (2008). Diversidad de Macroinvertebrados Acuáticos en un Nacimiento de Río en el Páramo de Santurbán, Norte de Santander. 32(122): 79-86.

Esteves, F. A. (1988). Fundamentos de Limnología. Ed. Interciencias. FINEP. Rio de Janeiro, Brasil. 578 p.

Giacometti, J. (2006). Macroinvertebrados Acuáticos y su Importancia como Bioindicadores de Calidad del Agua en el Río Alambi. Universidad Central, Ecuador. 2: 17-32.

González, V. (2012). Aplicación de los Índices de Calidad de Agua NSF, DINIUS y BMWP.

Hawkins, C. P., Murphy M. L., \& Anderson, N. H. (1982). Effects of canopy, substrate composition, and gradient on the structure of macroinvertebrate communities in Cascade Range streams of Oregon. Ecology, 63(6): 1840-1856.

Heino, J., Louhi, P. \& Muotka, T. (2004). Identifying the scales of variability in stream macroinvertebrate abundance, functional composition and assemblage structure. Freshw Biol. 49(9): 1230- 1239.

Little, C. \& Lara, A. (2010). Restauración ecológica para aumentar la provisión de agua como un servicio ecosistémico en cuencas forestales del centro-sur de Chile. Bosque, 175-178.

Lozano, L. (2005). La Bioindicación de la Calidad del Agua: Importancia de los Macroinvertebrados en la Cuenca Alta del Río Juan Amarillo, Cerros Orientales de Bogotá. Universidad Manuela Beltrán, Bogotá - Colombia. 
Luna, H. (2009). Estudio Preliminar del Uso de Macroinvertebrados Acuáticos como Bioindicadores de la calidad de Agua en la Quebrada Mamarramos y en un Sector del Río Cane en el Santuario de Fauna y Flora de Iguaque. Universidad Industrial de Santander, Bucaramanga - Colombia.

Margalef, R. (1983). Los organismos como indicadores en la limnología. Inst. Forestal Inv., 300.

Motomura, O. (2002). Ética, Vida, Sustentabilidad. Programa de las Naciones Unidas para el Medio Ambiente, Red de Formación Ambiental para América Latina y el Caribe, 5-331.

Ramírez, A., Pringle, C. \& Wantzen, M. (2008). Tropical Stream Conservation. En: Dudgeon, D. (Ed), Tropical Stream Ecology. Academic Press is an imprint of Elsevier. San Diego, California, USA.

Rey-Benayas, J., A Newton, A. Díaz \& J. Bullock. (2009). Enhancement of biodiversity and ecosystem services by ecological restoration: A Meta-Analysis. Science 325: 1121-1124.

Rocha-G, B. (2007). Evaluación de la calidad del agua a partir de bioindicadores e índices de calidad "caso de estudio: cuenca alta del río Chicamocha". Trabajo de grado Especialización en Ingeniería de Sistemas Hídricos Urbanos, Universidad de Los Andes.

Roldán, G. (1999). Los Macroinvertebrados y su valor como indicadores de la calidad del agua. Revista de la Academia Colombiana de Ciencias exactas, físicas, y naturales, 23(88): 375-387.

Roldán, G. (2003). Bioindicación de la calidad del agua en Colombia. Editorial Universidad de Antioquia. Colombia. 170 p.

Roldán, G. \& Ramírez, J. (2008). Fundamentos de limnología neotropical- 2. edición-.Editorial Universidad de Antioquia, Colombia. 4.

Romero, F. I., Cozano, M. A., Gangas, R. A., \& Naulin, P. I. (2014). Zonas ribereñas: protección, restauración y contexto legal en Chile. Bosque, 3-12.

Romero, F. (2014) Zonas Ribereñas: Protección, Restauración y Contexto Legal en Chile. Universidad de Chile.

Sistema Nacional de Parques Naturales. Plan de Manejo Santuario de Fauna y Flora Iguaque. (2006). Colombia. 63.

SER (Society for Ecological Restoration International-Sociedad Internacional para la Restauración Ecológica). (2004). Grupo de trabajo sobre ciencia y políticas. Principios de SER International sobre la restauración ecológica.

Terneus, E. \& Hernández, K. (2012). Evaluación Ecológica del Río Lliquino a Través de Macroinvertebrados Acuáticos, Pastaza - Ecuador. Universidad del Valle. 
Vannote, R., Minshall, G., Cummins, K., Sedell, J. \& Cushing, C. (1980). The River Continuum Concept. Aquat Sci. 37:130-137.

Vargas, O. (2007). Guía metodológica para la restauración ecológica del bosque altoandino. Bogotá.

Walteros, J. (2008). Estudio Preliminar de la Comunidad de Macroinvertebrados Acuáticos en la Reserva Forestal Torre Cuatro.

Zúñiga, M., Rojas, A. \& Caicedo, G. (1993). Indicadores ambientales de calidad de agua en la Cuenca del río Cauca. Asociación de Ingenieros Sanitarios de Antioquia Medellín, Colombia. 2: 17-28. 